\title{
A POSSIBLE NEW DIMENSION AT A FEW TEV
}

\author{
I. Antoniadis \\ Centre de Physique Théorique \\ Ecole Polytechnique-91128 Palaiseau,France \\ and \\ CERN, Geneva
}

\begin{abstract}
We examine the possibility of the existence of a large internal dimension at relatively low energies of the order of a few $\mathrm{TeV}$. Such dimension is a general prediction of perturbative string theories, which relate its size to the supersymmetry breaking scale. We point out that, contrary to our naive expectations, this senario is consistent with perturbative unification up to the Planck scale, in a particular class of "four"-dimensional string models. Furthermore, it has spectacular phenomenological consequences, whose main effects are discussed.
\end{abstract}

CPTH-A978.0690

June 1990 
A main problem in string phenomenology is the mechanism which breaks space-time supersymmetry. The latter is expected to occur at low energies, of the order of the electroweak scale, to protect the gauge hierarchy. Besides, this mechanism is crucial in string model bulding in order to destroy the flat directions and make definite predictions for the various masses and couplings. Surprisingly, despite the (infinitely) large degeneracy of four-dimensional string vacuua, the spontaneous breaking of supersymmetry at a scale $m_{s}$ much smaller than the Planck mass $M_{p}$ turns out to be a very hard problem, at least in perturbation theory $[1,2]$.

The first obvious question to ask is whether this scale could correspond to an arbitrary, at the tree level, continuous parameter, as is the case in ordinary supergravity. In string theories though, the only continuous parameters are vacuum expectation values (vev's) of scalar fields along flat directions of their potential. These, in principle, can not break spontaneously supersymmetry, since it is well known that the super-Higgs effect is not a usual Higgs phenomenon but needs non vanishing vev's for the auxiliary fields of the supermultiplets. In fact, space-time supersymmetry requires that the world-sheet action must have $\mathrm{N}=2$ superconformal invariance. The continuous breaking is then reduced to the problem of connecting smoothly $\mathrm{N}=2$ to $\mathrm{N}=12 \mathrm{~d}$ superconformal theories, which is shown to be impossible [2].

This result does not rule out the possibility of making $m_{s}$ small by tuning some discrete parameter. Furthermore, following some supergravity examples, one could have a large gravitino mass but still a small observable breaking scale, characterized for instance by the gaugino or scalar masses. This would amount again a "discrete fine-tuning", because in string theories all these masses are proportional: in the limit of massless gauginos the gravitino becomes massless, as well. The existence of such discrete parameter seems unlike, although there is no general proof.

The final possibility is that $m_{s}$ is related to some other continuous parameter of the theory, such as an internal compactification radius. In this case, the broken theory is continuously connected to the supersymmetric one, only in the decompactification limit which is infinitely far away. Some explicit examples of this kind have been worked out in the litterature $[3,4]$. There are several reasons to believe that this is the only way to obtain a small supersymmetry breaking scale in string perturbation theory. In fact, the problem can be studied in a general way in a large class of four-dimensional models, the so called "Gaussian", which are constructed using free bosons or free fermions on the worldsheet. It has been shown [1] that the scale $m_{s}$ can be small only if it is linked to the size of some internal dimension, which is decompactified to a scale roughly of the same order of magnitude. The same conclusion was also found in all other examples studied so 
far, realizing spontaneous supersymmetry breaking at the string level, using completely different methods [5].

Although there is no direct experimental contradiction, the possibility of having one or more large internal radii is found uninteresting for theoretical reasons [6]: a naive dimensional analysis shows that all couplings become huge very early above the extra dimension scale, invalidating the semi-classical string description.Moreover, various arguments have been given claiming that string theory has no acceptable weakly coupled vacuua [7].

Non perturbative effects are thus left as the final alternative to realize supersymmetry breaking. The popular senario is the gaugino condensation in a gauge group from the hidden sector [8]. The generated superpotential term for the dilaton field has not however critical points, unless one adds a constant contribution arising from the field strength of the antisymmetric tensor in the internal space [8] or from gravitino condensation [9]. Unfortunately, this constant is found to be quantized [10] of order one in Planck units, and the resulting breaking scale $m_{s}$ is very high. The situation can be improved when one includes the contribution of composite matter fields [11] or the effects of a condentation in a second group factor [12] from the hidden sector. The main disadvantage of this approach is that there is no framework for calculating non perturbative effects in the full string theory and one is forced to work in the effective field theory, making the assumption that this is a good approximation, and in any case to loose partially calculability.

The fact that the problem of supersymmetry breaking is so restrictive in string theories gives perhaps the hope that its solution could provide strong constraints for model bulding. Using the non perturbative approach, the effective theory at the end does not look very different from models one obtains in the supergravity context. Although its particular form is determined by string symmetries and most masses and couplings are in principle calculable under certain assumptions, there is no some definite low energy prediction which could be a direct consequence of the stringy character of the theory. The aim of this work is to study carefully the perturbative approach, which makes the spectacular prediction of the existence of an extra dimension at low energies, and examine if there is, and under which conditions, a way out of the resulting theoretical problems.

When supersymmetry is broken, one in general expects that radiative corrections will generate a non zero cosmological constant $\Lambda$, supressed by some low power of the breaking scale $m_{s}$. Even in a non perturbative senario if we achieve broken supersymmetry with zero vacuum energy at the tree level of the effective theory, there is no a priori reason to believe that $\Lambda$ will remain zero in higher orders. In string theory, the problem becomes more severe because a non zero $\Lambda$ generates also a non trivial potential for the dilaton and in general for all moduli fields, like those corresponding to the internal radii. Leaving appart 
the cosmological constant problem, in this paper we point out that there is a mechanism, in a class of $4 \mathrm{~d}$ string models, which breaks supersymmetry at the string level and does not spoil perturbative calculability, avoiding the problem of huge corrections in the coupling constants.

String theories realize consistently the old Kaluza-Klein idea of unifying gravity with gauge interactions in a higher dimensional theory and solves in particular one of its major problems, namely the appearence of chirality after the compactification in four dimensions. Moreover, in addition to the usual Kaluza-Klein (KK) exitations, they predict the existence of winding modes which give rise to new stringy-symmetries, the so called space-time dualities. These are discrete transformations which, in the simplest case, exchange the compactification radius $R$ to $1 / R$ (in Planck units) implying that small and large radii are completely equivalent.

Let us point out that in the field theory context a large internal dimension does not make any sense not only because of the coupling problem mentioned earlier, but mainly because the theory looses its predictability even at low energies, below the compactification scale. The reason is that above this scale it behaves like a higher dimensional non renormalizable theory, and the decoupling theorem does not apply for the massive KK modes; one in general needs to introduce an infinite number of low energy parameters. Recall that in any renormalizable theory, massive modes decouple at low energies after the redefinition of a finite number of couplings, to absorb the divergences which arise in the loops when their mass goes to infinity [13].

The situation is improved drastically in the context of string theory. The predictability of the theory is maintained as a consequence of the finiteness: all massive modes associated to the extra dimension decouple at low energies by redefining just one parameter, the coupling constant. In fact in the large radius limit, although the theory becomes effectively higher dimensional, string calculations provide finite results in terms of the higher $D$ dimensional coupling constant $g_{D}$ related to the four-dimensional one $g_{4}$ by

$$
g_{D}^{2}=R^{D-4} g_{4}^{2}
$$

where $R^{D-4}$ is the volume of the internal space. Of course, above the extra dimension scale, the effective field theory description breaks down and the full string theory has to be taken into account. However, despite decoupling is recovered calculability is in general lost, since the coupling constant grows very rapidly above the compactification scale and the theory becomes strongly interacting.

To be more explicit, let us concentrate on the internal radii dependence of loop effects in four dimensional string models with $\mathrm{N}=1$ space-time supersymmetry. By virtue of non 
renormalization theorems for the superpotential, the Yukawa-couplings remain unchanged. Putting apart the kinetic-terms, only the gauge couplings can in principle receive loop corrections which, at the one loop level, take the form [14]:

$$
\frac{1}{g_{i}^{2}(\mu)}=\frac{k_{i}}{g_{4}^{2}}+\frac{b_{i}}{4 \pi^{2}} \log \frac{M_{p}^{2}}{\mu^{2}}+\frac{1}{4 \pi^{2}} \Delta_{i}
$$

where $g_{i}^{2}(\mu)$ is the coupling constant of the $i$-th gauge group factor, realized as a level $k_{i}$ Kac-Moody algebra, depending on the energy scale $\mu ; b_{i}$ is the one loop beta-function and $\Delta_{i}$ the corresponding threshold effects. From the point of view of the effective $N=1$ supergravity, $1 / g_{i}^{2}\left(M_{p}\right)$ corresponds to the expectation value of a function $f$, which multiplies the kinetic terms of the gauge bosons:

$$
L_{\text {eff }}=-\frac{1}{4} \operatorname{Re} f F_{\mu \nu}^{2}+\frac{\imath}{4} \operatorname{Im} f F_{\mu \nu} \tilde{F}^{\mu \nu}
$$

$f$ is an analytic function of the chiral massless superfields at the tree level, but its analyticity properties are modified at higher orders [15]. String theory implies at lowest order that $f_{i}=k_{i} S$, where $S$ is the scalar component of the dilaton chiral supermultiplet: its real part is the dilaton field whose expectation value determines the string coupling constant $g_{4}=<\operatorname{Re} S>^{-1 / 2}$, while its imaginary part is related to the axion field which is the dual of the two-index antisymmetric tensor.The threshold corrections $\Delta_{i}$ have been computed recently in orbifold theories [16] following the method of Ref[14]. The internal radii dependence can also be derived by symmetry arguments based on space-time dualities [15]. It turns out that $\Delta_{i}$ receive radii-dependent corrections only from those sectors of the theory, which "preserve" exactly a $\mathrm{N}=2$ space-time supersymmetry.

In fact, consider a four-dimensional model obtained by a compactification of the tendimensional superstring on a $6 \mathrm{~d}$ torus $T^{6}$ divided by a discrete $S U(3)$ subgroup $H$, so that $\mathrm{N}=1$ space-time supersymmetry remains unbroken. The one loop partition function is written as a sum of sectors $\left(h_{\alpha}, h_{\beta}\right)$ over all elements of $\mathrm{H}$ with boundary conditions $h_{\alpha}$ and $h_{\beta}$ along the two cycles of the world-sheet torus. Each sector may preserve $N=4,2$ or 1 supersymmetries. The only sector which preserves $N=4$ is the untwisted one $(1,1)$; this does not give any contributions to the $\beta$-functions and to $\Delta_{i}$ because the corresponding integrand vanishes due to the summation over the fermionic spin structures [14], in a way analogous to the vanishing of the partition function. On the other hand, a $N=1$ "twisted" sector can provide only constant contributions, radii-independent, since the corresponding states do not carry any six-momenta or windings. They are left the $\mathrm{N}=2$ sectors, which correspond to boundary conditions rotating two out of the three complex planes of $T^{6}$, and give therefore contributions depending on the "moduli" of the third unrotated plane. 
The latter is characterized by four real parameters, namely the three components of the internal $2 \mathrm{~d}$ metric on the plane $G_{\alpha \beta}$ plus the antisymmetric tensor $B_{\alpha \beta}=\epsilon_{\alpha \beta} \frac{b}{\sqrt{G}}$, which are combined to form two complex fields $T=\frac{1}{2}(b+\imath \sqrt{G})$ and $\Sigma=\left(G_{12}+\imath \sqrt{G}\right) / G_{11}$ [17]. In the particular case one considers just a common internal radius $R$, these are reduced to $T=\frac{\imath}{2} R^{2}$ and $\Sigma=\imath$.

The corresponding threshold corrections $\Delta_{i}$ are $[16] \dagger$ :

$$
\Delta_{i}=-\hat{b}_{i} \operatorname{Re} \log \left[c T_{2} \eta^{4}(T) \Sigma_{2} \eta^{4}(\Sigma)\right]
$$

where $\eta(T)=q^{\frac{1}{24}} \prod_{n=1}^{\infty}\left(1-q^{n}\right)$ is the Dedekind-eta function with $q=e^{2 \imath \pi T}, T_{2}=\operatorname{Im} T$, $c$ a moduli-independent constant, and $\hat{b}_{i}$ the $\beta$-function of the corresponding $N=2$ sector of the model. $\mathrm{Eq}(4 \mathrm{a})$ is invariant under the duality symmetry $S L(2, \mathbf{Z}) \times S L(2, \mathbf{Z}) / \mathbf{Z}_{2}$, generated by the transformations $T \rightarrow-\frac{1}{T}, T \rightarrow T+1, \Sigma \rightarrow-\frac{1}{\Sigma}, \Sigma \rightarrow \Sigma+1$ and $T \leftrightarrow \Sigma$. These fix uniquely its form from its asymptotic behavior which is calculable at the level of the effective field theory [18]. For large radius eq(4a) becomes

$$
\Delta_{i} \sim \frac{\pi}{6} \hat{b}_{i} R^{2}-\hat{b}_{i} \log R^{2}+O(1)
$$

and gives huge corrections to the coupling constants in (2). The first term in (4b) can be understood as a consequence of the non-renormalizable character of the $\mathrm{N}=2$ part of the effective theory, which behaves as six-dimensional in the energy region below the Planck mass, up to the compactification scale. Its specific form can be fixed from the requirement that in the limit $R \rightarrow \infty$ the theory should give finite results, in terms of the six-dimensional coupling constant $g_{6}$ defined in $\mathrm{eq}(1)$ for $\mathrm{D}=6$. Moreover, the logarithmic term replaces essentially the ultraviolet cutoff $M_{p}$ in (2) by the compactification scale $R^{-1}$.

At this point we can address the crucial question, of whether there is a way out of the large coupling problem. The answer is yes if all $\Delta_{i}$ happen to be independent of some internal radius, which therefore can be made large without leaving huge corrections to the coupling constants. From the previous analysis, there are obviously two cases with the desired property:

There are firstly models which do not have $\mathrm{N}=2$ sectors at all, as for instance is the case of $\mathbf{Z}_{3}$ or $\mathbf{Z}_{7}$ orbifolds which break $N=4$ supersymmetry directly down to $N=1$. The fact that the corresponding threshold effects do not depend on the internal radii looks counter-intuitive from the point of view of the effective field theory, since above the decompactification scale the Kaluza-Klein excitations start contributing in the $\beta$-functions

$\dagger$ In general, there is an additional not a priori calculable contribution to $\Delta_{i}$ proportional to $k_{i}$ [14]. Since this provides a common correction for all group factors, it can be absorbed into a redefinition of $g_{4}$ and is irrelevant for our purposes. 
and change very rapidly the logarithmic behavior (2) to a power dependence, as expected in a non-renormalizable theory according to (4b) [18]. The explanation is based on the presence of extended $(\mathrm{N}=4)$ supersymmetry in the corresponding sectors of the theory, which leads to cancellations among the bosonic and fermionic loops, resulting a vanishing of $\beta$-functions and threshold corrections. Moreover, $\mathrm{N}=1$ sectors give only constant contributions because they do not depend on the internal radii, as explained previously.

On the other hand, one can imagine models with $\mathrm{N}=2$ sectors, but in which the moduli of some internal complex plane does not appear in the corrections $\Delta_{i}$. This could happen if for instance this plane is rotated by all boundary conditions, or if the corresponding $\beta$ functions $\hat{b}_{i}$ in (4) vanish for all group factors. Note that the latter does not a priori implies the vanishing of the actual $\beta$-functions of the theory $b_{i}$ in (2). Concerning higher loop contributions, there are indications that at least the asymptotic radius dependence of the one loop effects does not get renormalized, which are currently under investigation. These are related to some analogous known results in global supersymmetry, that $\beta$-functions vanish in $\mathrm{N}=4$, while they are given by their one loop expression in $\mathrm{N}=2$.

Although in $\mathbf{Z}_{3}$ orbifold the radius independence is automatic, technical difficulties related to the supersymmetry breaking will force us to study the case of $\mathbf{Z}_{2} \times \mathbf{Z}_{2}$. This will serve as the simplest non trivial illuminating example, which also appears in fermionic constructions and has promissing phenomenology. $\mathbf{Z}_{2} \times \mathbf{Z}_{2}$ is generated by the basis elements $h_{1}, h_{2}$ with $h_{1,2}^{2}=1$. Denoting the three internal compactified complex coordinates $\Phi_{\alpha}(\alpha=1,2,3)$, part of the action of $h_{1}, h_{2}$ and $h_{3} \equiv h_{1} h_{2}$ is to transform with a minus sign $\left(\Phi_{2}, \Phi_{3}\right),\left(\Phi_{1}, \Phi_{3}\right)$ and $\left(\Phi_{1}, \Phi_{2}\right)$, respectively. The one loop partition function is then splitted in three parts, characterized by the number of unbroken supersymmetries $\mathrm{N}=4,2$ or 1

$$
Z=\frac{1}{4}\left[\begin{array}{l}
1 \\
1
\end{array}\right]+\frac{1}{4} \sum_{\alpha=1}^{3}\left(\left[\begin{array}{c}
1 \\
h_{\alpha}
\end{array}\right]+\left[\begin{array}{c}
h_{\alpha} \\
1
\end{array}\right]+\left[\begin{array}{l}
h_{\alpha} \\
h_{\alpha}
\end{array}\right]\right)+\frac{1}{4} \sum_{\alpha \neq \beta}\left[\begin{array}{l}
h_{\alpha} \\
h_{\beta}
\end{array}\right]
$$

where $\left[\begin{array}{c}h \\ h^{\prime}\end{array}\right]$ is the contribution of a sector with boundary conditions $h$ and $h^{\prime}$ along the two cycles of the world-sheet torus. The periodic sector 1 is described by some appropriate momentum lattice of the six compactified coordinates, which in the absence of the twist defines a $\mathrm{N}=4$ theory denoted by $\left[\begin{array}{l}1 \\ 1\end{array}\right]$, while the factor $1 / 4$ counts for the $\mathbf{Z}_{2} \times \mathbf{Z}_{2}$ projection. $\mathrm{Eq}(5 \mathrm{a})$ can be rewritten as

$$
Z=-\frac{1}{2}\left[\begin{array}{l}
1 \\
1
\end{array}\right]+\frac{1}{2} \sum_{\alpha=1}^{3} Z_{\alpha}\left(R_{\alpha}\right)+\frac{1}{4} \sum_{\alpha \neq \beta}\left[\begin{array}{l}
h_{\alpha} \\
h_{\beta}
\end{array}\right]
$$

where $Z_{\alpha}\left(R_{\alpha}\right)=\frac{1}{2}\left(\left[\begin{array}{l}1 \\ 1\end{array}\right]+\left[\begin{array}{c}1 \\ h_{\alpha}\end{array}\right]+\left[\begin{array}{c}h_{\alpha} \\ 1\end{array}\right]+\left[\begin{array}{l}h_{\alpha} \\ h_{\alpha}\end{array}\right]\right)$ is the partition function of a $N=2$ theory depending on the moduli $R_{\alpha}$ of the complex plane $\Phi_{\alpha}$. 
The two projections break also the gauge group $G$ of the initial $\mathrm{N}=4$ theory to a product, in general, of four factors $G_{1} \times G_{2} \times G_{3} \times G_{4}$. However, each of the three $\mathrm{N}=2$ sectors preserve a bigger gauge symmetry $G_{14} \times G_{23}, G_{24} \times G_{13}$ or $G_{34} \times G_{12}$ for $h_{1}, h_{2}$ or $h_{3}$ respectively, where $G_{i j}$ is broken to $G_{i} \times G_{j}$ at the $\mathrm{N}=1$ level. The threshold corrections for each of the group factors are then given in eq(2) with $\Delta_{i}=\sum_{\alpha=1}^{3} \Delta_{i}^{\alpha}\left(R_{\alpha}\right)$ where $\Delta_{i}^{\alpha}$ are expressed in eq(4a). Simple inspection of (5b) shows that the corresponding coefficients $\hat{b}_{i}^{\alpha}=\frac{1}{2} b_{i}^{\alpha}$, where $b_{i}^{\alpha}$ is the one-loop $\beta$-function of the gauge coupling $g_{i}$ calculated in the $\alpha$-th sector of the theory. Moreover, $b_{i}^{\alpha}$ satisfy the relation

$$
\frac{1}{2} \sum_{\alpha=1}^{3} b_{i}^{\alpha}=b_{i}
$$

which through eq(4b) implies that in the case of a large common radius $R$, the ultraviolet cutoff $M_{p}$ in (2) is replaced by the compactification scale $R^{-1}$. $\mathrm{Eq}(6)$ shows that the vanishing of $b_{i}^{\alpha}$ for every $i$ in some $\alpha$-th $\mathrm{N}=2$ sector of the theory, does not in general implies the vanishing of the $\beta$-functions $b_{i}$.

Up to now we pointed out the existence of $N=1$ string models, whose effective couplings do not depend on some internal radius $R$. The next step is to use this radius in order to break supersymmetry spontaneously at the string level, in a scale $m_{s} \sim 1 / R$. The resulting theory can thus have a small $m_{s}$ for large $R$ without loosing perturbative calculability. In fact, the $R$-dependence of threshold corrections in the broken theory is expected to be suppressed, at least by powers of $m_{s} / M_{p}$ or equivalently $1 / R$.

Let us first describe the spontaneous supersymmetry breaking mechanism. We will employ the method of "twisted tori" or "coordinate dependent compactifications" which extends the "Scherk-Schwarz" mechanism in string theory $[3,4]$. The idea is to couple the lattice momenta of toroidal compactifications to the charges of a $U(1)$ current, which does not commute with the gravitino vertex operator and therefore breaks supersymmetry. This is achieved without destroy modular invariance by boosting the corresponding momenta of the "Lorentzian" lattice, in analogy with the spontaneous breaking of gauge symmetries [19]. In the resulting theory all masses sre shifted according to the "Lorentz-boost", while the amount of shifts depends on the momenta and the $U(1)$-charges of the states, as well as on the parameter of the transformation.

Consider, for instance, the case of one internal coordinate $\phi=\operatorname{Re} \Phi_{1} \equiv\left(\phi_{L} ; \phi_{R}\right)$ compactified on a torus of radius $R$ with momenta

$$
p_{L, R}=\frac{m}{R} \pm \frac{n R}{2}
$$

and one real $U(1)$ left-moving current $J=\partial \varphi$, and apply the $S O(2,1)$ transformation of 
their corresponding momenta $P \equiv\left(p, p_{L} ; p_{R}\right)[3,4]$

$$
P \rightarrow P e^{\Omega} \quad \text { with } \quad \Omega=\left(\begin{array}{ccc}
0 & \xi & \xi \\
-\xi & 0 & 0 \\
\xi & 0 & 0
\end{array}\right)
$$

where $\xi=\frac{\omega}{R}$ and $\Omega^{3}=1$. Using (7), eq(8a) gives

$$
\begin{aligned}
p & \rightarrow p-\omega n \\
n & \rightarrow n \\
m & \rightarrow m+\omega p-\frac{\omega^{2}}{2} n
\end{aligned}
$$

and breaks supersymmetry, since the gravitino, which carries zero-momentum and winding numbers $m=n=0$, gets a mass $M_{3 / 2}=\frac{\omega p}{\sqrt{2} R}$ proportional to its $U(1)$-charge $p$. Varying the parameter $\omega$ of the transformation (8), the broken theory could be continuously connected to the supersymmetric one $\omega=0$. However $\omega$ can not be arbitrary, because the current $J$ does not commute with the world-sheet supercurrent $T_{F}$, as it has to transform non trivially the space-time gravitino. The only allowed values of $\omega$ are those which generate discrete transformations $e^{22 \pi \omega} \int J$ commuting with $T_{F}$. A last restriction is that $J$ must transform in the same way as $\phi$ under all boundary conditions. For instance in the $\mathbf{Z}_{2} \times \mathbf{Z}_{2}$ example, $J$ must be, as $\phi$, periodic under $h_{1}$ and antiperiodic under $h_{2}$. In the antiperiodic (twisted) sector, the corresponding momenta $P$ are zero and there are no mass-shifts.

Trying to apply this method to the $\mathbf{Z}_{3}$-orbifold, we found unfortunately no example of a current $J$ with the desired properties, which can be used to break supersymmetry. It is not clear if this is a technical problem related to the particular method, or if there is some deeper reason. Restricting ourselves to the $\mathbf{Z}_{2} \times \mathbf{Z}_{2}$ case, consider as an example a model with the property that in the first $\mathrm{N}=2$ subsector $Z_{1}$ in (5b), the corresponding $\beta$-functions $b_{i}^{1}$ vanish for all group factors $i \nmid$. All coupling constant corrections are then independent of the moduli of the first complex plane $\Phi_{1}$, which we treat for simplicity just as one common radius $R$. A possible choice for the left $U(1)$-current $J$ is $J=f_{2}^{R} f_{3}^{R}$ [4], where $f_{\alpha}^{R} \equiv \operatorname{Re} f_{\alpha}$ and $f_{\alpha}$ are the complex $2 \mathrm{~d}$ fermionic superpartners of $\Phi_{\alpha}$, so that the world-sheet supercurrent $T_{F}$ is

$$
T_{F}=\psi^{\mu} \partial X_{\mu}+\frac{1}{2} \sum_{\alpha=1}^{3}\left(f_{\alpha}^{\star} \partial \Phi^{\alpha}+\text { h.c. }\right) .
$$

$\dagger$ A more general constraint is sufficient to be satisfied: $\sum_{\alpha=1}^{3} \varepsilon_{\alpha} \hat{b}_{i}^{\alpha} \Delta\left(R_{\alpha}\right)=0$ for every $i$, where $\hat{b}_{i}^{\alpha} \Delta\left(R_{\alpha}\right)=\Delta_{i}^{\alpha}\left(R_{\alpha}\right)$ defined in $(4 \mathrm{a})$ and $\varepsilon=0$ or 1 . 
$J$ has clearly the same periodicity properties as $\operatorname{Re} \Phi_{1}$, under all boundary conditions, and one can apply transformation (8) to break spontaneously space-time supersymmetry. The parameter $\omega$ must be chosen equal to one, which corresponds to a $2 \pi$-rotation of the $2 \mathrm{~d}$ fermions $f_{2,3}^{R}$ leaving invariant the supercurrent (9).

However, the effect of transformation (8) is not trivial, as one can conclude by considering for instance the states with zero winding number $(n=0)$. There are two categories of such states: Those which correspond to $f_{2,3}^{R}$ antiperiodic have integer charges $p$ and remain invariant under $(8 \mathrm{~b})$, up to a trivial relabeling $m \rightarrow m-p$. On the other hand, the states which correspond to $f_{2,3}^{R}$ periodic transform in the spinorial representation and change sign under a $2 \pi$-rotation. Hence, they have half-integer charges $p$ and, up to a relabeling, their masses are shifted by

$$
\delta M^{2}=\frac{1}{8 R^{2}}+\frac{m}{2 R^{2}} .
$$

In particular, the previously massless states of this category acquire a common mass equal to $\frac{1}{\sqrt{8} R}$. These are the gravitino, all fermions of the $\mathrm{N}=2$ vector supermultiplets which become the gauginos, and all scalars of $N=1$ chiral multiplets which emerge from the $\mathrm{N}=2$ hypermultiplets of the $Z_{1}$ subsector of the theory (5). Note that the pattern of supersymmetry breaking depends on the choice of the current $J$. Although a complete analysis of the various possibilities has not been performed, a study of Ref[4] in the context of this mechanism shows that this is the only choice which can avoid the appearence of tachyons with odd soliton number for some range of values of the radius $R$.

One can now calculate the radius dependence of the one loop corrections $\Delta_{i}^{1}(R)$ to the coupling constants, in the spontaneously broken theory. Following Ref[14], these are expressed as an integral over the modulus $\tau$ of the world-sheet torus, restricted in the fundamental domain $\Gamma \equiv\left\{\tau_{2}>0,\left|\tau_{1}\right|<\frac{1}{2},|\tau|>1\right\}$ :

$$
\Delta_{i}^{1}(R)=\int_{\Gamma} \frac{d^{2} \tau}{\tau_{2}}\left\{|\eta(\tau)|^{-4} \sum_{\vec{a}, \vec{b}} c\left(\begin{array}{l}
\vec{a} \\
\vec{b}
\end{array}\right) \frac{d Z_{\psi}}{2 \imath \pi d \tau} Z^{(2,1)}(R) \operatorname{Tr}\left(Q_{i}^{2} q^{H} \bar{q}^{\bar{H}}\right)_{\mathrm{int}}^{\mathrm{rest}}-b_{i}^{1}\right\}
$$

where $\vec{a}, \vec{b}$ are vectors of boundary conditions for the world-sheet fermions with $a=1$ or 0 denoting periodic or antiperiodic $2 \mathrm{~d}$ fermion, and the coefficients $c\left(\frac{\vec{a}}{\vec{b}}\right)$ are signs determined the GSO (fermion-number) projections. The contribution of the two transverse fermionic coordinates $\psi^{\mu}$ is given by $Z_{\psi}\left(\begin{array}{c}a_{\psi} \\ b_{\psi}\end{array}\right)=\frac{\Theta\left(\begin{array}{c}a_{\psi} \\ b_{\psi}\end{array}\right)}{\eta}$, while $Z^{(2,1)}$ stands for the contribution of the two-dimensional torus corresponding to the internal coordinate $\Phi_{1}$, as well as for the contribution of the $U(1)$ current $J$. Finally, in the trace of the remaining part of the theory $Q_{i}^{2}$ selects only the states which are non-singlets under the gauge group factor $G_{i}$. 
In the supersymmetric theory, $Z^{(2,1)}$ reads

$$
Z_{s}^{(2,1)}\left(\begin{array}{l}
a \\
b
\end{array}\right)=Z_{T}^{2}(R) \frac{\Theta\left(\begin{array}{l}
a \\
b
\end{array}\right)}{\eta}
$$

where $Z_{T}(R)$ is the torus partition of radius $R: Z_{T}=\frac{1}{|\eta|^{2}} \sum_{p_{L}, p_{R}} q^{\frac{1}{2} p_{L}^{2}} \bar{q}^{\frac{1}{2} p_{R}^{2}}$, with momenta $p_{L, R}$ given in (7). From the $\Theta$-function expression in (12a) one finds that the $U(1)$-charges are given by $p=k+\frac{a}{2}$ with $k$ integer. The broken theory is obtained by applying the transformation (8) with $\omega=1$. Redefining $k \rightarrow k+n$ and $m \rightarrow m-k$, one obtains:

$$
Z_{b}^{(2,1)}\left(\begin{array}{l}
a \\
b
\end{array}\right)=Z_{T}(R) \frac{\Theta\left(\begin{array}{l}
a \\
b
\end{array}\right)}{\eta} \sum_{n, m}(-)^{n b} q^{\frac{1}{2} p_{L}{ }^{\prime 2}} \bar{q}^{\frac{1}{2} p_{R}{ }^{\prime 2}}
$$

where $p_{L, R}^{\prime}$ are the transformed torus momenta, given in eq(7) with $n^{\prime}=n$ and $m^{\prime}=$ $m+\frac{a+n}{2}$. After a Poisson resummation in $m$, one finds the following expression for the difference $\delta Z^{(2,1)}$ between the contributions of the broken $\left(Z_{b}\right)$ and the unbroken $\left(Z_{s}\right)$ theory:

$$
\delta Z^{(2,1)}\left(\begin{array}{l}
a \\
b
\end{array}\right)=\frac{R}{\sqrt{2 \tau_{2}}} Z_{T}(R) \frac{\Theta\left(\begin{array}{l}
a \\
b
\end{array}\right)}{\eta} \frac{1}{|\eta|^{2}} \sum_{n, m} e^{-\frac{\pi R^{2}}{2 \tau_{2}}|m+n \tau|^{2}}\left[(-)^{n m+m a+n b}-1\right] .
$$

In the large radius limit, only the states with zero-winding number contribute $(n=0)$, and the only non vanishing contribution is

$$
\delta Z^{(2,1)}\left(\begin{array}{l}
1 \\
0
\end{array}\right) \sim-\frac{R^{2}}{\tau_{2}} \frac{1}{|\eta|^{4}} \frac{\Theta_{2}}{\eta} \sum_{m} e^{-\frac{\pi R^{2}}{2 \tau_{2}}(2 m+1)^{2}}
$$

up to terms which give exponentially suppressed corrections for large $R$ in the integral (11). Using (13b) into eq(11) and changing variables from $\tau_{2}$ to $x=\tau_{2} / R^{2}$, one finds that $\delta \Delta_{i}^{1}$ goes to a constant:

$$
\delta \Delta_{i}^{1}(R) \sim b_{i}^{s^{1}}+O\left(e^{-\lambda R^{2}}\right)
$$

where $b_{i}^{s^{1}}$ is the contribution to the $\beta$-function $b_{i}^{1}$ of the fields with half-integer $U(1)$ charges, whose masses are therefore shifted by the transformation (8). Eq(14) shows that there are no large loop corrections in the broken theory. In fact, up to a constant, these are exponentially suppressed in terms of the supersymmetry breaking scale $m_{s}^{2} \sim 1 / R^{2}$.

The radius dependence of the one loop cosmological constant can also be derived, if one substitutes eq(13) into the partition function. The latter has an expression similar to (11), obtained by omitting $Q_{i}^{2}$ from the trace and replacing $\frac{d Z_{\psi}}{d \tau}$ with $\frac{1}{r_{2}^{2}} Z_{\psi}$. The same change of variables which provided eq(14) now gives:

$$
\Lambda^{(1-\mathrm{loop})} \sim\left(n_{B}-n_{F}\right) \frac{1}{R^{4}}+O\left(e^{-\lambda R^{2}}\right)
$$


where $n_{B}-n_{F}$ is the difference between the number of bosons and fermions remained massless after supersymmetry breaking. The factor $1 / R^{4}$ gives a power suppression similar to the one obtained from $\mathrm{N}=4$ supergravity, but unfortunately not sufficient to push $\Lambda$ below its experimental upper limit. It remains however the possibility of being exponentially supressed, if $n_{B}=n_{F}[20]$.

In conclusion, in this work we point out the possibility of the existence of some internal dimension at relatively low energies, of the order of a few $\mathrm{TeV}$. It is motivated by perturbative string theory, which necessarily relates the supersymmetry breaking scale to the size of this internal dimension. Such a senario is theoretically unacceptable in ordinary field theories because of their non renormalizable character in higher dimensions, which invalidates the decoupling of the Kaluza-Klein excitations and destroyes perturbative calculability very rapidly above the compactification scale. However, we argued that, in the context of some class of "four"-dimensional string models, it is perfectly consistent with perturbative grand-unification up to the Planck scale. This is due to a combination of the stringy character of the theory together with the presence of an extended supersymmetry in the relevant sector, which leads to the appropriate cancellations in the $\beta$-functions. The effects of such extra dimensions are spectacular and give two main sets of direct predictions, which are detectable in the present and next generation of experiments:

(i) Predictions related to the decompactification phenomenon of these dimensions at the $\mathrm{TeV}$ range. The main signal is the regular repetition of states with exactly the same quantum numbers, which are the ordinary KK-excitations with masses $\frac{m}{R}$ for $m=1,2,3$... corresponding to the fifth "quasicontinuous" component of the momentum. However, only a part of the states "feel" the extra dimension and have repetitions; these are all gauge particles, i.e. gauge bosons and gauginos, as well as some matter fields provided by the corresponding $\mathrm{N}=2$ hypermultiplets. This is a purely stringy phenomenon, related to the orbifold character of these models, which is also responsible for the appearence of chirality after the compactification. Only the "untwisted" fields have repetitions. In the "twisted" sectors the string center of mass is located on the orbifold fixed-points and their excitations do not "feel" the extra dimensions, since they do not carry corresponding momenta.

(ii) Predictions for the pattern of supersymmetry breaking which is strongly correlated to the compactification phenomenon. At the lowest order, the supersymmetry breaking is restricted to all multiplets which "feel" the extra dimension. The precise numbers are slightly model dependent and a more detailed analysis is required. We give here the results for the $\mathbf{Z}_{2} \times \mathbf{Z}_{2}$ orbifold in the context of the framework described earlier. The gravitino, gauginos and the scalar components of all chiral $\mathrm{N}=1$ supermultiplets which have repetitions, acquire a common mass $\frac{1}{\sqrt{8} R}$. In the rest of the theory, supersymmetry 
breaking will be communicated through radiative corrections. A preliminary analysis then shows that an internal radius $R \sim 2-3 \mathrm{TeV}$ is consistent with all present experimental data and implies, in the context of the proposed mechanism, a spectrum of various superpartners of the known particles closed to the $\mathrm{TeV}$ region.

These are the primary consequences. A number of problems require however a more careful and detailed analysis. The most important are related to the puzzle of the cosmological constant and to the dynamical determination of the possible hierarchically large size of such extra dimension(s). Moreover, particular effort should be made to apply the proposed senario to some "realistic" string model, and relate the breaking of supersymmetry to the breaking of the standard model gauge group of electroweak interactions.

I would like to thank S. Dimopoulos and C. Kounnas for very useful discussions at the early stage of this work. 


\section{References}

[1] I. Antoniadis, C. Bachas, D. Lewellen and T. Tomaras, Phys. Lett. $207 \mathrm{~B}$ (1988) 441.

[2] T. Banks and L. Dixon, Nucl. Phys. B307 (1988) 93.

[3] R. Rohm, Nucl. Phys. B237 (1984) 553; C. Kounnas and M. Porrati, Nucl. Phys. B310 (1988) 355; S. Ferrara, C. Kounnas, M. Porrati and F. Zwirner, Nucl. Phys. B318 (1989) 75.

[4] C. Kounnas and B. Rostand, Ecole Normal preprint LPTENS 89/22 (1989).

[5] S.P. de Alwis, J. Polchinski and R. Schimmrigk, Phys. Lett. 218 B (1989) 449.

[6] V.S. Kaplunovsky, Phys. Rev. Lett. 55 (1985) 1036.

[7] M. Dine and N. Seiberg, Phys. Rev. Lett. 55 (1985) 366.

[8] J.P. Derendinger, L.E. Ibanez and H.P. Nilles, Phys. Lett. 155B (1985) 65; M. Dine, R. Rohm, N. Seiberg and E. Witten, Phys. Lett. 156B (1985) 55.

[9] K. Konishi, N. Magnoli and H. Panagopoulos, Nucl. Phys. B309 (1988) 201.

[10] R. Rohm and E. Witten, Ann. Phys. 170 (1986) 454.

[11] I. Antoniadis, J. Ellis, A.B. Lahanas and D.V. Nanopoulos, Phys. Lett. 241B (1990) 24.

[12] N.V. Krasnikov, Phys. Lett. 193B (1987) 37; L. Dixon, SLAC preprint SLAC-PUB5229 (1990); L. Dixon, V.S. Kaplunovsky, J. Louis and M. Peskin, to appear.

[13] T. Appelquist and J. Carazzone, Phys. Rev. D11 (1975) 2856.

[14] V.S. Kaplunovsky, Nucl. Phys. B307 (1988) 145.

[15] S. Ferrara, UCLA preprint UCLA/90/TEP/20 (1990); S. Ferrara, N. Magnoli, T.R. Taylor and G. Veneziano, CERN preprint CERN-TH.5744/90 (1990).

[16] L. Dixon, V.S. Kaplunovski and J. Louis, to appear.

[17] R. Dijkgraaf, E. Verlinde and H. Verlinde, in "Copenhagen 1987, Proceedings, Perspectives in String Theory".

[18] T.R. Taylor and G. Veneziano, Phys. Lett. 212B (1988) 147.

[19] K.S. Narain, Phys. Lett. 169B (1986) 41; S. Ferrara, C. Kounnas and M. Porrati, Nucl. Phys. B304 (1988) 500; I. Antoniadis, C. Bachas and C. Kounnas, Phys. Lett. 200B (1988) 297.

[20] H. Itoyama and T.R. Taylor, Phys. Lett. 186B (1987) 129. 